\title{
The quest for stable circumbinary companions to post-common envelope sdB eclipsing binaries ${ }^{\star}$
}

\section{Does the observational evidence support their existence?}

\author{
D. Pulley, G. Faillace, D. Smith, A. Watkins, and S. von Harrach
}

British Astronomical Association, Burlington House, Piccadilly, London W1J 0DU, UK

e-mail: david@davidpulley.co.uk; binaryfaillace@aol.com

Received 8 May 2017 / Accepted 3 November 2017

\begin{abstract}
Context. Period variations have been detected in a number of eclipsing close compact binary subdwarf B stars (sdBs) and these have often been interpreted as being caused by circumbinary massive planets or brown dwarfs. According to canonical binary models, the majority of sdB systems are produced from low mass stars with degenerate cores where helium is ignited in flashes. Various evolutionary scenarios have been proposed for these stars, but a definite mechanism remains to be established. Equally puzzling is the formation of these putative circumbinary objects which must have formed from the remaining post-common envelope circumbinary disk or survived its evolution.

Aims. In this paper we review the eclipse time variations (ETVs) exhibited by seven such systems (EC 10246-2707, HS 0705+6700, HS 2231+2441, J08205+0008, NSVS 07826147, NSVS 14256825, and NY Vir) and explore whether there is conclusive evidence that the ETVs observed over the last two decades can reliably predict the presence of one or more circumbinary bodies.

Methods. We report 246 new observations of the seven sdB systems made between 2013 September and 2017 July using a worldwide network of telescopes. We combined our new data with previously published measurements to analyse the ETVs of these systems. Results. Our data show that period variations cannot be modelled simply on the basis of circumbinary objects. This implies that more complex processes may be taking place in these systems. These difficulties are compounded by the secondary star not being spectroscopically visible. From ETVs, it has historically been suggested that five of the seven binary systems reported here had circumbinary objects. Based on our recent observations and analysis, only three systems remain serious contenders. We find agreement with other observers that at least a decade of observations is required to establish reliable ephemerides. With longer observational baselines it is quite conceivable that the data will support the circumbinary object hypothesis of these binary systems. Also, we generally agree with other observers that higher values of $(\mathrm{O}-\mathrm{C})$ residuals are found with secondary companions of spectral type M5/6 (or possibly earlier as a result of an Applegate type mechanism).
\end{abstract}

Key words. binaries: close - binaries: eclipsing - planetary systems - planets and satellites: formation - subdwarfs

\section{Introduction}

Since 2007, from observations of eclipse time variations (ETVs), there have been many claims made for the detection of circumbinary objects around post-common envelope subluminous eclipsing binary systems. These sdB systems are members of the HW Vir family of short period binary systems that consist of a very hot sdB type star and a cool, low mass, main sequence star or brown dwarf companion. Their compact structures and the large temperature differences between the two components give rise to short and well-defined primary eclipses allowing times of minima to be determined with high precision. The sdB component of these systems have canonical masses of $\sim 0.47 M_{\odot}$ and consist of a helium burning core with a thin hydrogen envelope; they are located on the left side of the horizontal branch in the Hertzsprung-Russell diagram.

Various evolutionary scenarios have been proposed for these stars, but a definitive mechanism remains to be established, in particular whether or not binary evolution, as outlined by Paczynski (1976), Webbink (1984), and Zorotovic et al. (2010),

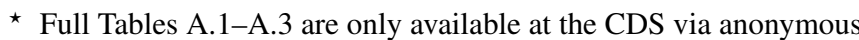
ftp to cdsarc.u-strasbg. fr (130.79.128.5) or via http://cdsarc.u-strasbg.fr/viz-bin/qcat?]/A+A/611/A48
}

is a requirement. These models suggest that when the more massive primary evolves, it fills its Roche lobe during one of the red giant phases and unstable mass transfer from the primary occurs at a rate that cannot be accommodated by the secondary component. This results in material forming a non-corotating common envelope that surrounds the core of the red giant and the secondary component, thus enshrouding the binary system. Angular momentum is transferred from the binary system to the common envelope bringing the binary pair closer together and resulting in a short binary period of typically between 2 and $3 \mathrm{~h}$. Eventually the common envelope has sufficient orbital energy to overcome its binding energy and is mostly ejected from the system on a timescale of $\sim 1000 \mathrm{yr}$ (Xiong et al. 2017), initially creating a protoplanetary nebula and then a planetary nebula leaving a stellar remnant well on its way to becoming a white dwarf. The short duration of the common envelope phase means the mass of the secondary companion is assumed to remain constant and the mass of the remaining compact object, the primary, would be effectively equal to the mass of the core of the red giant at the onset of mass transfer.

Zorotovic \& Schreiber (2013) provided an overview of 13 of these systems. Interestingly, in 5 of the 13 systems, ETVs have been interpreted as showing the presence of low mass 
Table 1. Summary of the seven objects observed.

\begin{tabular}{|c|c|c|c|c|c|c|c|c|}
\hline Object & $\begin{array}{c}\text { RA } \\
\text { J2000 }\end{array}$ & $\begin{array}{c}\text { Dec } \\
\text { J2000 }\end{array}$ & $\begin{array}{l}\text { Period } \\
\text { (days) }\end{array}$ & $\begin{array}{l}\text { Distance } \\
\mathrm{kpc}\end{array}$ & Mag & $\begin{array}{l}\text { Pri } \\
\text { min }\end{array}$ & $\begin{array}{l}\mathrm{Sec} \\
\mathrm{min}\end{array}$ & $\begin{array}{l}\text { Observing } \\
\text { Period }\end{array}$ \\
\hline EC 10246-2707 & 102656.472 & -272257.11 & 0.118507985 & - & $14.77(\mathrm{~V})$ & 7 & 0 & 2016 Jan.-2017 Jan. \\
\hline HS $0705+6700$ & 071042.056 & +66 5543.52 & 0.095646609 & 1.7 & $14.60(\mathrm{R})$ & 101 & 16 & 2013 Sep.-2017 May \\
\hline HS $2231+2441$ & 223421.483 & +245657.39 & 0.110587829 & 0.17 & $14.20(\mathrm{~V})$ & 26 & 3 & 2014 Dec.-2017 Jan. \\
\hline $\mathrm{J} 08205+0008$ & 082053.076 & +000842.78 & 0.096240737 & 0.61 & $15.17(\mathrm{~V})$ & 33 & 6 & 2014 Dec.-2017 Feb. \\
\hline NSVS 07826147 & 153349.446 & +375928.06 & 0.1617704491 & 0.6 & $13.08(\mathrm{~V})$ & 20 & 0 & 2015 Apr.-2017 Jun. \\
\hline NSVS 14256825 & 202000.475 & +043756.49 & 0.1103741681 & 0.6 & $13.34(\mathrm{R})$ & 19 & 0 & 2015 Aug.-2017 Jul. \\
\hline NY Vir & 133848.142 & -020149.24 & 0.1010159668 & 1.1 & $13.30(\mathrm{~V})$ & 15 & 0 & 2015 Apr.-2017 Jun. \\
\hline
\end{tabular}

Notes. A total of 246 times of minima have been determined, of which 221 were primary minima.

circumbinary objects (e.g. brown dwarfs, massive planets). If such bodies do exist, then they must have either survived the energetic common envelope ejection process or have formed during this short period from the remnant of the ejected common envelope. From simulations, Zorotovic \& Schreiber (2013) concluded that the latter was the most likely scenario, the so-called second generation hypothesis. Supporting this view, Schleicher \& Dreizler (2014) concluded that the formation of planets from the ejected common envelope seemed feasible. Using primarily the NN Ser post-common envelope eclipsing binary system model, which has confirmed circumbinary planets (Parsons et al. 2014; Hardy et al. 2016), they estimated the mass loss and the fraction of mass that remains gravitationally bound to the system. This remaining mass would form a protoplanetary disk, resulting in new planets. In contrast, Bear \& Soker (2014), based on angular momentum considerations, proposed a firstgeneration hypothesis where existing planets survive the energetic common envelope ejection process.

In this paper we present the eclipse time variations exhibited by the seven sdB systems and analyse the results in the context of the circumbinary planet hypothesis. The seven systems studied are listed in Tables 1 and 2 .

\section{Observing method and data reduction}

We report 246 new observations of the seven sdB systems made between 2013 September and 2017 July using the telescopes and filters listed in Tables A.1 and A.2. The effects of differing atmospheric extinctions were minimised by making all observations at altitudes greater than $40^{\circ}$. All images were calibrated using dark, flat, and bias frames and then analysed with MaxIm DL software $^{1}$. The source flux was determined with aperture photometry using a variable aperture, whereby the radius is scaled according to the full width at half maximum (FWHM). Variations in observing conditions were accounted for by determining the flux relative to a comparison star in the field of view. Apparent magnitudes, coordinates, and separation from the target for each comparison star are given in Table A.3.

When using filters the apparent magnitude of the target was derived from the apparent magnitudes of the comparison stars and the average magnitude of the target calculated by the software. The comparison stars' catalogue magnitudes for the various filters were taken from the American Association of Variable Star Observers (AAVSO) Photometric All Sky Survey (APASS) catalogue, and were similar to the target magnitudes. An attempt was made to select comparison stars with similar colour indices to the target stars, although this proved difficult given the very blue nature of the sdB systems. Because the APASS catalogue

\footnotetext{
1 MaxIm DL, http://www.diffractionlimited.com/
}

does not include the $R$ pass band, in the few cases where observations were taken with the $R$ filter a conversion formula recommended by AAVSO was used to transform the catalogue Sloan $r^{\prime}$ magnitudes to the corresponding $R$ magnitudes. When observations were performed without filters, check stars were used to ensure that there was no variability in the reference star selected. A summary of the various target systems investigated together with their basic properties are listed in Tables 1 and 2. A detailed analysis of each system is provided in the following sections.

All of our new timings used in this analysis were first converted to barycentric Julian date dynamical time (BJD_TBD) using the time utilities of the Ohio State University ${ }^{2}$. We then calculated the times of minima using the Kwee \& van Woerden (1956) procedure coded in the Peranso ${ }^{3}$ software package and cross-checked these results with both Kwee and van Woerden and the Fourier procedures of the Minima ${ }^{4}$ software package. In one instance, EC 10246-2707 at JD 2457408.6459, we only had observations around the minimum and through the egress. Here we fitted an inverted Gaussian curve, with parameters derived from a good light curve, minimising the residuals and deriving a time of minimum which was in very good agreement with the expected time of minimum.

Our new timings were combined with previously published times of minima and, where appropriate, the historic times were converted to BJD_TBD before computing a new linear and/or quadratic ephemerides and residuals. Where a new linear or quadratic ephemeris was calculated only observed primary minima data were used. We also excluded data that used phase folded or synthetic light curve techniques. These techniques are frequently employed in data analysis of wide field sky surveys.

The difference between the observed and calculated times of minima $(\mathrm{O}-\mathrm{C})$ can be used to infer potential internal or external influences on the binary pair, for example (i) angular momentum loss through magnetic braking or the emission of gravitational waves; (ii) angular momentum redistribution through Applegate-type mechanisms; (iii) the apparent changing of the binary period through the presence of a circumbinary object; or (iv) apsidal motion (see e.g. Brinkworth et al. 2006; Bours et al. 2016, and references therein).

\section{Analysis of eclipse timings}

The $(\mathrm{O}-\mathrm{C})$ diagrams for each system shown in this section include both primary and secondary minima together with data

\footnotetext{
2 The Ohio State University,

http://astroutils . astronomy . ohio-state.edu/time/

3 Peranso, http: //www . peranso.com/

4 Nelson B., Minima, https://www.variablestarssouth.org/ software-by-bob-nelson/
} 
D. Pulley et al.: The quest for stable circumbinary companions to post-common envelope sdB eclipsing binaries

Table 2. Summary of key parameters of the binary systems observed.

\begin{tabular}{|c|c|c|c|c|c|c|c|c|c|c|c|}
\hline Object & $\begin{array}{c}M_{1} \\
\left(M_{\odot}\right)\end{array}$ & $\begin{array}{c}M_{2} \\
\left(M_{\odot}\right)\end{array}$ & $\begin{array}{c}\text { incl } \\
\text { (degs) }\end{array}$ & $\begin{array}{l}T_{\text {eff }} \\
(\mathrm{K})\end{array}$ & $\begin{array}{c}a \\
\left(R_{\odot}\right)\end{array}$ & $\begin{array}{l}\text { Sp. Type } \\
\left(M_{2}\right)\end{array}$ & $\begin{array}{c}\text { RMS } \\
\text { (Residuals) }\end{array}$ & $\log g$ & $\begin{array}{c}K_{1} \\
\left(\mathrm{~km} \mathrm{~s}^{-1}\right)\end{array}$ & $\log \left(\frac{n_{\mathrm{He}}}{n_{\mathrm{H}}}\right)$ & Reference \\
\hline EC 10246-2707 & 0.45 & 0.12 & 80.0 & 28900 & 0.84 & M5/M6 & 1.60 & 5.64 & 71.6 & -2.50 & Barlow et al. (2013) \\
\hline HS $0705+6700$ & 0.48 & 0.13 & 84.4 & 28800 & 0.81 & M4/M5 & 19.89 & 5.40 & 85.8 & -2.68 & Drechsel et al. (2001) \\
\hline HS $2231+2441$ & 0.265 & 0.05 & 79.1 & 28370 & 1.18 & $\mathrm{BD}$ & 2.47 & 5.39 & 49.1 & -2.91 & Ostensen et al. (2008) \\
\hline $\mathrm{J} 08205+0008$ & $\begin{array}{l}0.25 \\
/ 0.47\end{array}$ & $\begin{array}{l}0.045 \\
/ 0.068\end{array}$ & 85.9 & 26700 & $\begin{array}{l}0.6 \\
/ 1.1\end{array}$ & $\mathrm{BD}$ & 1.13 & 5.48 & 47.4 & & Geier et al. (2011) \\
\hline NSVS 07826147 & 0.376 & 0.113 & 86.6 & 29230 & & M5 & 2.41 & 5.58 & 71.1 & & For et al. (2010) \\
\hline NSVS 14256825 & $\begin{array}{l}0.35 \\
/ 0.46\end{array}$ & $\begin{array}{l}0.097 \\
/ 0.21\end{array}$ & 82.5 & $\begin{array}{l}42300 \\
135250\end{array}$ & 0.74 & M5/M7 & 20.95 & $\begin{array}{l}5.50 \\
15.49\end{array}$ & 73.4 & $\begin{array}{l}-2.52 \\
/-2.70\end{array}$ & $\begin{array}{l}\text { Almeida et al. (2012) } \\
\text { Kilkenny \& Koen (2012) }\end{array}$ \\
\hline NY Vir & 0.39 & 0.11 & 80.7 & 31300 & 0.72 & M5 & $4.78 / 36.45$ & 5.74 & 78.6 & -2.91 & Vuckovic et al. (2007) \\
\hline
\end{tabular}

Notes. The spectral types for these systems are not clearly defined, being indirectly determined from light curve parameters which themselves can be poorly constrained. The RMS of residuals for NY Vir is shown with two values. Utilising all data the value is 36.45 , but eliminating the two Vuckovic data points, which Lee et al. (2014) reports with very low uncertainties, yields a value of 4.78.

from wide field sky surveys such as the Northern Sky Variability Survey (NSVS). SuperWASP data have been included where there are significant gaps in the historic data; when included we used the same binning methodology as Lohr et al. (2014). Error bars are shown where timing uncertainties are included in published data and do not affect the readability of the diagram.

In the following subsections, we present a brief historical review of seven $\mathrm{sdB}$ eclipsing binary systems and review the hypotheses presented by earlier observers, followed by an analysis of our new results. All our new times of minima for these systems are listed in Table A.2.

\subsection{EC 10246-2707}

\subsubsection{Background}

EC 10246-2707 was identified as an sdB star as part of the Edinburgh-Cape Faint Blue Object Survey (Kilkenny et al. 1997) and was discovered, during follow-up observations, to be an eclipsing binary system with a period of $\sim 2.8 \mathrm{~h}$. Simultaneously, and independently, Barlow identified the binary nature of the star. A joint University of North Carolina/Edinburgh-Cape paper was published (Barlow et al. 2013) reporting 49 times of minima and embracing spectroscopic and light curve analysis together with a linear ephemeris covering $15 \mathrm{yr}$ of observations. They determined the period of the binary as 0.1185079936 days and identified this system as a typical member of HW Vir family with $T_{1 \text { eff }}=28900 \mathrm{~K}, \log g=5.64, M_{1}=0.45 M_{\odot}$, and a likely $\mathrm{M}$ dwarf companion of mass $0.12 M_{\odot}$. They explored the possibility of a varying binary period, but found no statistical evidence to support this as opposed to a constant period.

Kilkenny (2014) provided ten new primary minima timings between 2013 and 2014 using the $1 \mathrm{~m}$ telescope at the South African Astronomical Observatory. He calculated a new linear ephemeris:

$T_{\min , \mathrm{BJD}}=2450493.46727(3)+0.1185079951(7) * E$.

As noted by Kilkenny (2014) the small change in residuals around mid-2012 lends support to a time varying period:

$$
\begin{aligned}
T_{\min , \mathrm{BJD}}= & 2450493.46733(3)+0.118507985(3) * E \\
& +2.0(5) * 10^{-13} * E^{2} .
\end{aligned}
$$

Kilkenny (2014) also showed that the effect of gravitational radiation on binary period reduction in HW Vir like systems is only detectable, with present equipment accuracies, after approximately a century and so could not explain these observed short period changes.

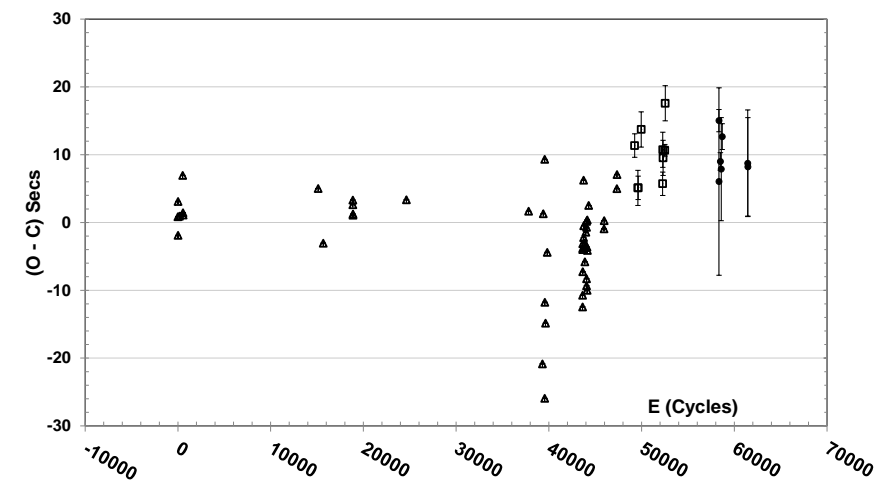

Fig. 1. (O-C) diagram for EC 10246-2707 using the Kilkenny (2014) linear ephemeris with our new data shown as solid dots, the Kilkenny (2014) data as open squares, and the Barlow et al. (2013) data as open triangles.

Application of the Mann-Whitney $U$ test (to identify whether the ranked absolute values of residuals from the linear and the quadratic ephemerides could come from the same parent population) indicates, at the $p=0.05$ level, that the linear ephemeris does not provide a statistically better fit to the data than the quadratic ephemeris. More data providing a longer timebase will verify which ephemeris is more applicable.

\subsubsection{Recent data and ephemerides}

We made seven observations between 2016 January and 2017 January using the $0.43 \mathrm{~m}$ iTelescope T17 Siding Spring, the $1 \mathrm{~m}$ Cerro Tololo, and the $1 \mathrm{~m}$ Sutherland telescopes. Our results integrate well with earlier data from Kilkenny (2014) and confirm an apparent change in binary period.

With our new data, together with published historical data, we recalculated both the linear and quadratic ephemerides, adopting the same reference epoch as used in Kilkenny (2014). Since we found little difference between these ephemerides and our derived ephemerides, we continue to use Kilkenny's ephemerides. The (O-C) diagrams with our new data are shown in Figs. 1 and 2 for the linear and quadratic ephemerides respectively. The linear fit of Fig. 1 does indicate a small binary period increase over the $20 \mathrm{yr}$ observational time span, but this increase appears to commence at $E \sim 47000$, corresponding to mid-2012. 


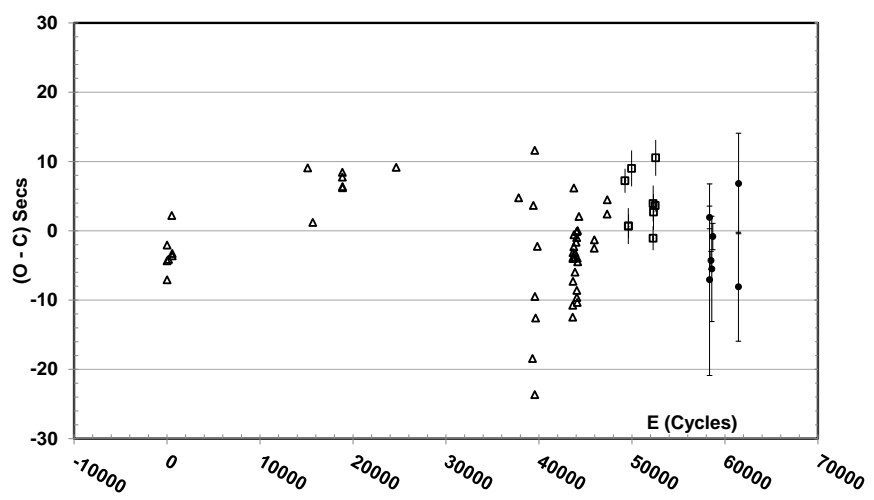

Fig. 2. (O-C) diagram for EC 10246-2707 using the Kilkenny (2014) quadratic ephemeris with our new data shown as solid dots, the Kilkenny data as open squares, and the Barlow and Kilkenny (2013) data as open triangles.

To quantify the goodness of fit, we determine the RMS of the weighted residuals (sometimes referred to as the reduced $\chi^{2}$ ) defined as

$\mathrm{RMS}=\sqrt{\frac{1}{n} \sum_{i}^{n}\left(\frac{O_{i}-C_{i}}{\sigma_{i}}\right)^{2}}$,

where $O_{i}, C_{i}$, and $\sigma_{1}$ are respectively the observed, calculated, and uncertainty in eclipse time at cycle $i$ taken over the $n$ datasets. For the linear and quadratic ephemerides the RMS computes to 3.02 and 1.60 , respectively.

Also of note is the wide spread in residuals, approximately $35 \mathrm{~s}$, at $E \sim 39500$, which is much greater than the typical error bars associated with each minimum. To a lesser degree this can also be seen at other times, e.g. $E \sim 44000$. More data will be required to establish the underlying trends.

\section{2. $H S 0705+6700$}

\subsubsection{Background}

HS 0705+6700 was identified as a $14.7 \mathrm{mag} \mathrm{sdB}$ star from the Hamburg Schmidt Quasar Survey. Follow-up observations by Drechsel et al. (2001) showed this to be a short period, $\sim 2.3 \mathrm{~h}$, post-common envelope eclipsing binary system; they produced the first linear ephemeris for this system. From their light curve and radial velocity measurements they calculated the mass and radius of the primary and secondary components to be $0.483 M_{\odot}$ and $0.239 R_{\odot}$ and $0.134 M_{\odot}$ and $0.186 R_{\odot}$, respectively. The orbital semi-major axis and inclination were determined as $0.81 R_{\odot}$ and $84.4^{\circ}$, respectively. The effective temperature of the sdB component was estimated to be $28800 \pm 900 \mathrm{~K}$. From these values they concluded the close companion was a cool $\mathrm{M}$ dwarf star contributing significant amounts of phase dependant reflected light.

Whilst Niarchos et al. (2003) and Çamurdan et al. (2012) provided corroborating light curve parameters, most investigators (Qian et al. 2009, 2010, 2013; Çamurdan et al. 2012; Beuermann et al. 2012; Pulley et al. 2015) have focused on an apparent quasi-periodic variation in the eclipsing binary period. Although not proven, and following the rejection of other possible mechanisms, e.g. orbital period modulation (Applegate 1992) or apsidal motion, the most likely cause of this variation has been attributed to the presence of a circumbinary object. A summary of the findings of these investigators is presented in Table 3.
Qian et al. (2010) predicted a period of $15.7 \mathrm{yr}$ for a circumbinary object in a circular orbit around the binary system. However, their Eq. (2) suggests that this is a typographical error and most probably the period should read $7.15 \mathrm{yr}$. They also found that the residuals could be further minimised with the introduction of a negative quadratic term into the ephemeris which, they suggested, could indicate the presence of a second circumbinary object. Çamurdan et al. (2012) identified a third light in their light curve analysis which, they believed, supported the circumbinary object hypothesis. However, neither they nor Beuermann et al. (2012) could find evidence to support a longterm period decrease.

Data from Qian et al. (2013) and Pulley et al. (2015) extended the timeline of the apparent circumbinary object into its second orbit and both preferred the inclusion of a positive quadratic term in the ephemeris.

\subsubsection{Recent data and ephemeris}

In the Addendum to Pulley et al. (2015) it was noted that a further 27 measurements taken between 2014 October and 2015 April showed a small departure from the predicted sinusoid of the third body. Insufficient data had been gathered to confirm the validity and the significance of this result, but we can now report a further 61 times of primary minima and four secondary minima observed between 2015 October and 2017 May.

From an analysis of all known times of minima between 2000 February and 2014 April, we published an unweighted quadratic ephemeris of

$$
\begin{aligned}
T_{\min , \mathrm{BJD}}= & 2451822.76155(5)+0.095646609(4) * E \\
& +5.5(9) * 10^{-13} * E^{2}+\tau_{3} .
\end{aligned}
$$

The cyclical light travel time effect, $\tau_{3}$, is given by the equation of Irwin (1952),

$$
\tau_{3}=\frac{a_{12} \sin i}{c}\left[\left(1-e^{2}\right) \frac{\sin (v+\omega)}{1+e \cos v}+e \sin \omega\right],
$$

where the projected semi-major axis, $a_{12} \sin i$, is 0.177 ; orbital eccentricity, $e$, is 0.03 ; longitude of periastron, $\omega$, is 0.119 ; and $v$ is the true anomaly. Full details can be found in Table 4 of Pulley et al. (2015). Using the quadratic portion of the above ephemeris we compute an RMS value of the weighted residuals of 19.89 .

We have computed the $(\mathrm{O}-\mathrm{C})$ residuals for our new data $(E>57000)$ and plotted these against the quadratic portion of the ephemeris of Eq. (4), as shown in Fig. 3. The cyclical nature of the residuals computed from Eq. (5) is shown by the sinusoidal curve in Fig. 3. We can confirm that the observations post $E=55000$ ( 2015 February) depart from predicted values as first reported in the Addendum to our 2015 paper. This effect can be clearly seen in Fig. 4 where the residuals have been computed after removing both the quadratic and cyclical effects described by Eq. (4).

The cyclical fit pre $E=55000$ is thought by many to be compelling evidence of a circumbinary object. Recent observations suggest that this system is entering a phase where $(\mathrm{O}-\mathrm{C})$ residuals are offset by $\sim 40 \mathrm{~s}$ from the original ephemeris. This change is indicative that the original circumbinary hypothesis is no longer applicable. More data are needed to see whether new circumbinary parameters can be computed for this system. An Applegate-type magnetic effect might provide an alternative explanation for the observed light travel time (LTT) effects. 
D. Pulley et al.: The quest for stable circumbinary companions to post-common envelope sdB eclipsing binaries

Table 3. Summary of observations and third body orbital parameters reported by previous observers for HS $0705+6700$.

\begin{tabular}{lcccccccc}
\hline \hline \multicolumn{1}{c}{ Parameter } & $\begin{array}{c}\text { Drechsel } \\
\text { et al. } \\
(2001)\end{array}$ & $\begin{array}{c}\text { Niarchos } \\
\text { et al. } \\
(2003)\end{array}$ & $\begin{array}{c}\text { Qian } \\
\text { et al. } \\
(2009)\end{array}$ & $\begin{array}{c}\text { Qian } \\
\text { et al. } \\
(2010)\end{array}$ & $\begin{array}{c}\text { Carmurdan } \\
\text { et al. } \\
(2012)\end{array}$ & $\begin{array}{c}\text { Beuermann } \\
\text { et al. } \\
(2012)\end{array}$ & $\begin{array}{c}\text { Qian } \\
\text { et al. } \\
(2013)\end{array}$ & $\begin{array}{c}\text { Pulley } \\
\text { et al. } \\
(2015)\end{array}$ \\
\hline Radial velocity analysis & Yes & No & No & No & No & No & No & No \\
Light curve analysis & Yes & Yes & No & No & Yes & No & No & No \\
Primary eclipse & 13 & 3 & 33 & 12 & 6 & 19 & 73 & 40 \\
Secondary eclipse & 0 & 3 & 5 & 2 & 0 & 3 & 5 & 12 \\
Binary ephemeris & & & & & & & & \\
\hline Binary period (days) & 0.09564665 & as Dreschel & 0.095646625 & 0.095646684 & 0.095564665 & 0.0956466253 & 0.095946611 & 0.0956466012 \\
Quadratic term (days) & - & - & - & $-1.87 \times 10^{-12}$ & - & - & $2.57 \times 10^{-12}$ & $1.52 \times 10^{-12}$ \\
Circumbinary Period (yr) & - & - & 7.15 & 15.7 & 8.06 & 8.41 & 8.87 & 8.73 \\
Eccentricity & - & - & 0 & 0 & not given & 0.38 & 0.19 & 0.22 \\
Semi-amplitude (s) & - & - & 92.4 & 71 & 98.5 & 86 & 87.4 & 80.1 \\
Min mass 3rd body $\left(M_{J}\right)$ & - & - & 39.5 & 30.4 & 37.7 & 29.6 & 32 & 33.2 \\
\hline
\end{tabular}

Notes. The circumbinary period reported by Qian et al. (2010) should probably read $7.15 \mathrm{yr}$. We think this is a typographical error.

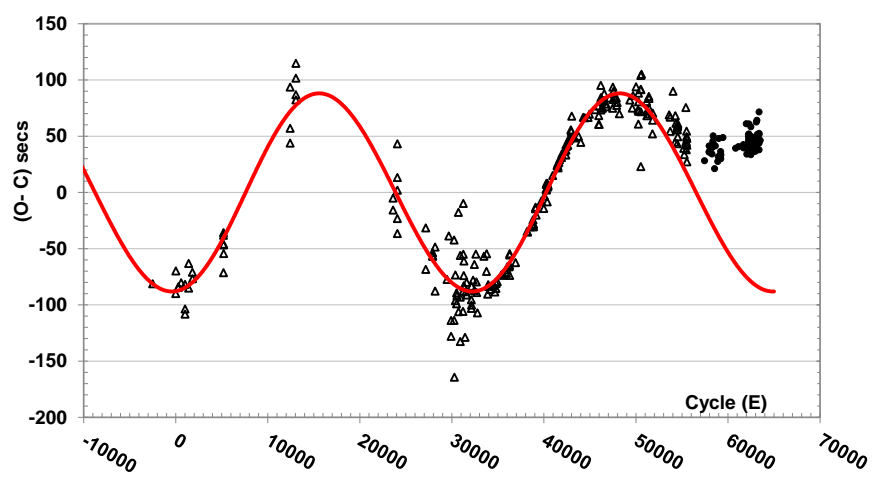

Fig. 3. (O-C) residuals for HS $0705+6700$ after removing the linear and quadratic terms: (i) open triangles display previously published data and (ii) solid dots show new data reported in this paper. Data have not been weighted.

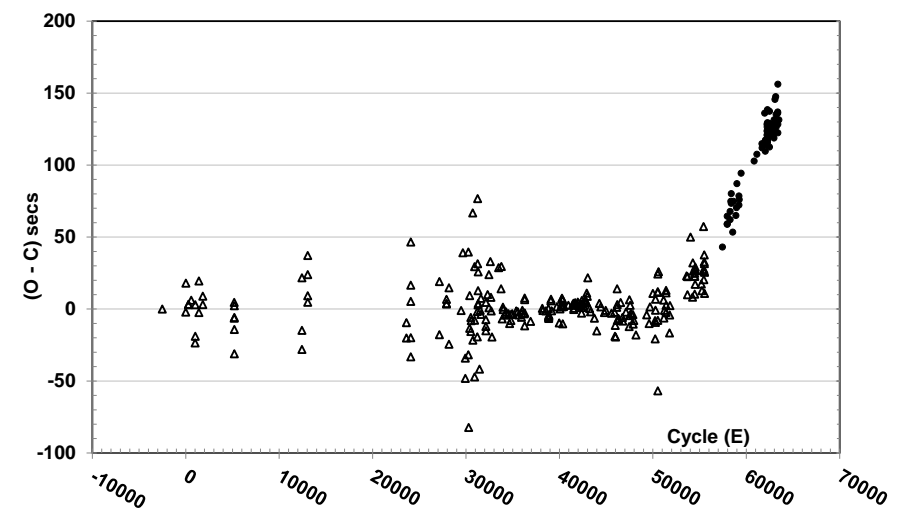

Fig. 4. (O-C) residuals for HS $0705+6700$ after removing the linear, quadratic and sinusoid terms: (i) open triangles display previous published data and (ii) solid dots show new data reported in this paper. Data have not been weighted.

These results undermine the earlier third body hypothesis. To a large extent, the original circumbinary hypothesis is based upon a dearth of data between 2002 and 2007 with data pre-2009 showing a degree of scatter $(1 \sigma \sim 25 \mathrm{~s})$ that is much larger than would be expected from a third body alone. This point remains unresolved.

\subsection{HS $2231+2441$}

\subsubsection{Background}

The discovery paper for HS $2231+2441$ was published by Østensen et al. (2007); it was described as a 14.5 mag short period, $\sim 2.7 \mathrm{~h}$, detached eclipsing binary system, thought to consist of a post-main sequence sdB star with a low mass dwarf $\mathrm{M}$ companion. Their analysis assumed a canonical mass of the primary of $0.47 M_{\odot}$, so helium burning. From spectroscopic measurements, Østensen et al. (2008) noted a significant inconsistency between the mass, radius, and surface gravity of the primary and revised down the primary mass from its canonical value to $0.265 M_{\odot}$ with a radius of $0.164 R_{\odot}$, thus placing the primary as a post-RGB object rather than an assumed core helium burning extreme horizontal branch star. With a mass ratio of 0.16 , the secondary becomes a substellar companion. Almeida et al. (2014) published a conference paper suggesting that the primary component was of even lower mass, $0.190 M_{\odot}$, but notably recording the semi-amplitude radial velocity of the primary as $37.8 \mathrm{~km} \mathrm{~s}^{-1}$, approximately $20 \%$ lower than that recorded by Østensen et al. (2007). A final confirmation of the true nature of this system would require the spectral signature originating from the secondary component.

Qian et al. (2010) described observations of the system's period between 2005 and 2009, and fitted a quadratic and a sinusoidal term to the $(\mathrm{O}-\mathrm{C})$ residuals. They suggested that the decrease in orbital period is associated with magnetic braking, and that a tertiary companion, with an orbital period of $4.76 \mathrm{yr}$, is responsible for the "apparent" cyclical variation. Using the absolute parameters from Østensen et al. (2007) they calculated the minimum mass of the tertiary companion as $0.0133 M_{\odot}$.

Although neither Qian nor $\varnothing$ stensen have published their times of minima, Lohr et al. (2014) extracted the Qian et al. (2010) timings from their Fig. 4. They also derived eclipse timings for this system from archival SuperWASP data. Lohr et al. (2014) noted that the SuperWASP data fitted Qian's circumbinary hypothesis well during 2006 and 2007, but did not strongly support the fit outside this date range, suggesting that a linear function might provide a better fit to the full data set.

\subsubsection{Recent data and ephemerides}

We have observed 26 primary minima and three secondary minima between 2014 December and 2017 January; these data are shown in the residuals plot in Fig. 5, where we have reproduced 


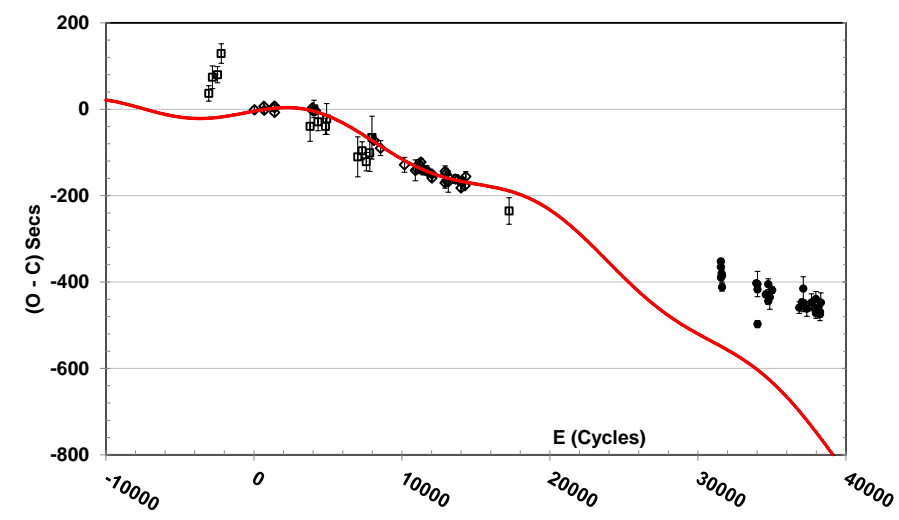

Fig. 5. Residuals for HS 2231+2441 using Ostensen's linear ephemeris Black open diamonds indicate the deconstructed data from Qian et al. (2010), open squares are data from SuperWASP, and solid circles are our latest data shown. The solid line shows Qian's quadratic ephemeris with the cyclical behaviour of a possible circumbinary object.

Fig. 4 from Qian et al. (2010), but with an extended timebase. This diagram shows their unpublished data which we recovered by deconstructing their $(\mathrm{O}-\mathrm{C})$ diagram, the SuperWASP data from Lohr et al. (2014) grouped into 30-day bins, and our recent data. The uncertainties introduced through deconstructing the Qian et al. (2010) data are estimated to be \pm 70 periods and \pm 0.00005 days.

As noted by Lohr et al. (2014), their early and late SuperWASP data are not in good agreement with the Qian et al. (2010) circumbinary hypothesis and they concluded that a linear ephemeris without a circumbinary component is more appropriate. Our new data, with minima occurring about $150 \mathrm{~s}$ before Qian's predictions, support the conclusions of Lohr et al. (2014).

Using the deconstructed data from Qian et al. (2010) and our new data we have calculated a linear ephemeris and a quadratic ephemeris over a time span of $12 \mathrm{yr}$ and find

$$
\begin{aligned}
T_{\min , \mathrm{BJD}}= & 2453522.66963(6)+0.110587855(2) * E \\
T_{\min , \mathrm{BJD}}= & 2453522.66980(9)+0.110587829(11) * E \\
& +6.46(3) * 10^{-13} * E^{2} .
\end{aligned}
$$

The Mann-Whitney $U$ test was used to compare the $(\mathrm{O}-\mathrm{C})$ residuals of the linear ephemeris with those of the quadratic ephemeris, and the null hypothesis was upheld. We conclude that there is no significant difference in the magnitudes of the two sets of residuals and thus no preference for either ephemeris. The calculated RMS of the weighted residuals also showed similar values of 2.47 and 2.37 for the linear and quadratic ephemerides, respectively. Thus, we have opted for the simpler linear ephemeris where the plot of the residuals is shown in Fig. 6 and where we have put the SuperWASP data back in. Whilst the linear fit looks appropriate, the data scatter appears comparable to the measurement uncertainties in the data and could indicate the presence of other factors.

\subsection{J082053.53+000843.4}

\subsubsection{Background}

$\mathrm{J} 082053.53+000843.4$, hereafter $\mathrm{J} 08205$, is a 15.2 mag short period detached eclipsing binary system. Like other systems described in this paper, J08205 belongs to a subclass of the HW Vir family of very short period, $\sim 2.3 \mathrm{~h}$, eclipsing binary

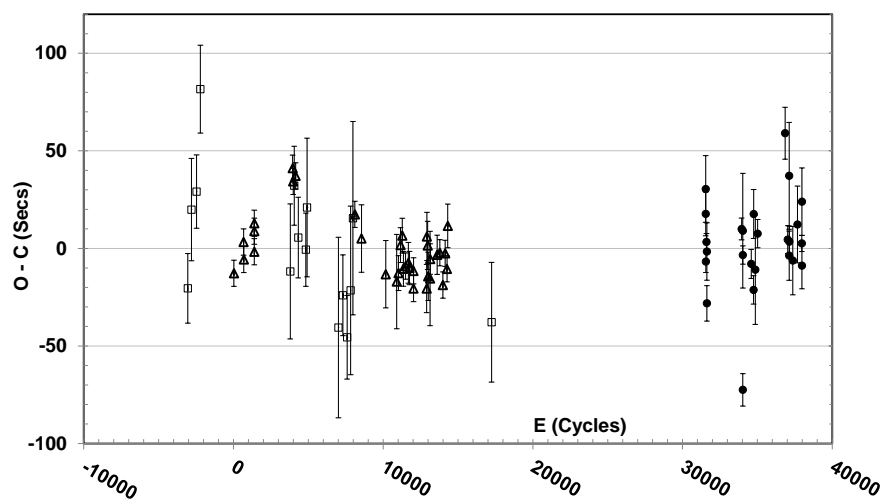

Fig. 6. (O-C) residuals for HS $2231+2441$ derived from the new linear ephemeris of Eq. (6). The deconstructed Qian data are shown as open triangles; our new data are shown as solid circles. The binned SuperWASP data are shown as open squares.

systems comprising a post-main sequence hot subdwarf star $(\mathrm{O}-$ or B-type) and most probably a brown dwarf companion.

J08205 was identified as an eclipsing sdB binary by Geier et al. (2011) as part of the Massive Unseen Companions to Hot Faint Underluminous Stars from SDSS (MUCHFUSS) project. Further publications by Schaffenroth et al. (2011) and Geier et al. (2012) focused on identifying the system parameters from both spectroscopic and light curve analyses.

Prior to 2016 April there had been no published eclipse timings for this system and, consequently, no indication as to whether there was any variation in the binary period. Pulley et al. (2016b) provided the first comprehensive set of eclipse timings for this system and laid the foundation for exploring the presence of a potential third body in a system where magnetic quadrupole effects generated by the companion brown dwarf are expected to be much reduced or non-existent.

\subsubsection{Recent data and ephemeris}

We recorded 19 sets of primary minima and 6 secondary minima collected between 2014 December and 2015 February. Details of these data and our analysis were published in Pulley et al. (2016b). Subsequently, we recorded 14 additional primary minima between 2015 November and 2017 February. These times of minima, together with the 20 earlier primary minima, allowed the computation of a system ephemeris spanning 26 months of recorded data. We refined this further with the inclusion of an unpublished minimum from Schaffenroth extending the measurement timebase back to 2012 January. This improved the precision of the calculated binary period by two orders of magnitude.

We explored the linear and the quadratic ephemerides and found that the Mann-Whitney $U$ test identified no significant difference in the magnitude of the residuals between them. Likewise, there was no significant difference in values of RMS weighted residuals of 1.127 and 1.131 and so we have opted for a new linear ephemeris:

$T_{\text {min,BJD }}=2457016.61105(2)+0.096240737(7) * E$.

This ephemeris has been used to calculate the $(\mathrm{O}-\mathrm{C})$ residuals plotted in Fig. 7. From this analysis there is no clear evidence of a systematic change in binary period. If quasi-cyclical LTT effects are present, they are most probably restricted to a semiamplitude of no greater than $20 \mathrm{~s}$. Whilst these results suggest the binary period is unchanging, confirmation will require further observations. 


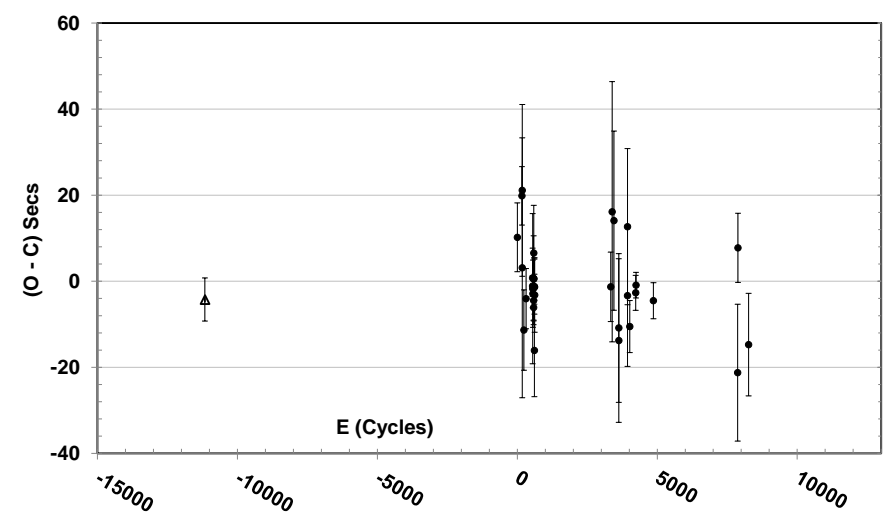

Fig. 7. (O-C) residuals for J08205+0008 with our new data grouped between $3000<E<8000$. The single open triangle represents Schaffenroth's single data point of 2012 January.

\subsection{NSVS 07826147}

\subsubsection{Background}

NSVS 07826147 was first identified as a short period, $\sim 3.9 \mathrm{~h}$, eclipsing sdB binary with an M2 or later main sequence companion by Kelley \& Shaw (2007). Spectroscopic analysis by For et al. (2010) suggested that the sdB had a mass lower than its canonical value together with an eclipsing M5 companion. They fitted a linear ephemeris to 21 times of minima observed between 2008 February and 2009 March. Zhu \& Qian (2010) produced 16 additional times of minima between 2009 March and 2009 August. Their revised period for this system fell within the measurement uncertainty of that predicted by For et al. (2010). Backhaus et al. (2012) determined a further seven times of minima between 2011 February and 2011 October and included a 2005 February observation from Drake et al. (2010), but noted that the uncertainty of this result is two orders of magnitude greater than their uncertainties. As a consequence we have not used Drake's result in our analysis. Lohr et al. (2014) provided additional timings from SuperWASP extending the timeline back to 2004 May.

Zhu et al. (2015b) were first to claim detecting a cyclical change in the period of this system; this was later confirmed by Zhu et al. (2015a), who reported that this periodic change could be caused by a circumbinary object of mass greater than $4.7 M_{J}$ with an orbital radius of 0.64 AU introducing an LTT effect of $\sim 0.00004$ days $(\sim 3.5 \mathrm{~s})$.Neither publication states a period, but Zhu et al. (2015a) Fig. 2 suggests $\sim 11000$ cycles, equivalent to $\sim 4.9 \mathrm{yr}$.

\subsubsection{Recent data and ephemeris}

We report a further 20 times of primary minima observed between 2015 April and 2017 June. These new data points together with the 35 previously published observed times of primary minima extend the timeline to over $13 \mathrm{yr}$. We explored both a linear and a quadratic ephemeris and found that the Mann-Whitney $U$ test identified no significant difference in the magnitude of the residuals between the two ephemerides. Likewise there was no difference in values of RMS weighted residuals and so opted for a new linear ephemeris:

$T_{\min , \mathrm{BJD}}=2455611.92655(1)+0.161770449(2) * E$.

This ephemeris is similar to that of Backhaus et al. (2012). With the new linear ephemeris, the computed $(\mathrm{O}-\mathrm{C})$ residuals are

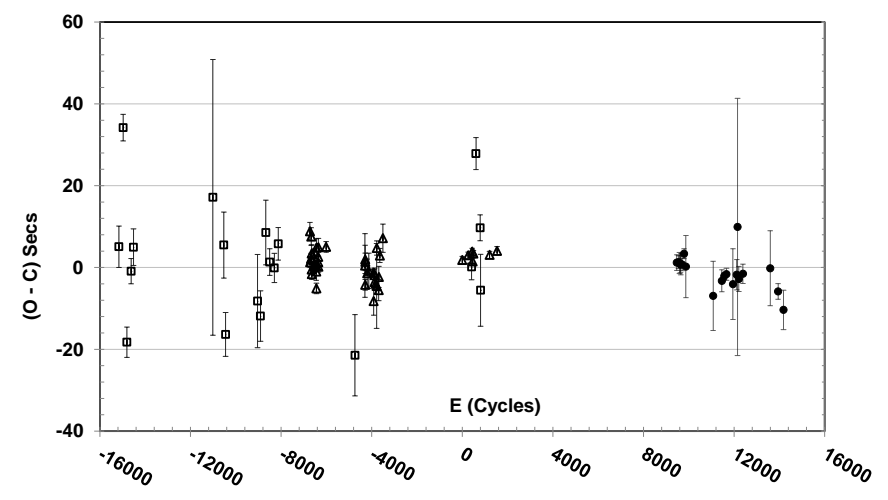

Fig. 8. (O-C) residuals for NSVS 07826147 . The historical data from For, Zhu, and Backhaus shown as open triangles; SuperWASP binned data as open squares and our new data represented as solid circles.

shown in Fig. 8 where the diagram also includes the 19 binned data sets from SuperWASP, Lohr et al. (2014), and nine observed secondary minima recorded by For et al. (2010). The RMS of the weighted residuals was calculated to be 2.41 .

The plot of $(\mathrm{O}-\mathrm{C})$ residuals shows some degree of scatter with a $\sigma \sim 10 \mathrm{~s}$ but there is no strong indication of any longterm change in the binary period. Zhu et al. (2015a) indicated the presence of a circumbinary object with an LTT of $\sim 3.5 \mathrm{~s}$, which is much less than the large scatter in the residuals $(\sim 10 \mathrm{~s})$. As yet they have not published any new data or analysis supporting their hypothesis so that drawing these conclusions may be premature.

\subsection{NSVS 14256825}

\subsubsection{Background}

NSVS 14256825 was discovered as a 13.2 mag variable star in the NSVS survey (Woźniak et al. 2004). Wils et al. (2007) identified this object as an eclipsing binary system with an sdB primary component having an orbital period of $\sim 2.6 \mathrm{~h}$. From their measurements, they produced V, B, and Ic light curves and documented 21 primary eclipses over a three-month period. They determined an ephemeris and suggested this object was similar in many respects to both the prototype $\mathrm{sdB} / \mathrm{M}$ dwarf system $\mathrm{HW}$ Vir and HS 0705+6700.

Qian et al. (2010) suggested that the binary period of NSVS 14256825 had a cyclical change, but did not provide any supporting evidence. Kilkenny \& Koen (2012) provided a further nine times of minima and showed that the orbital period was increasing at a rate of $\sim 1.2 \times 10^{-12}$ days per orbit. Beuermann et al. (2012) presented additional eclipse timings and their analysis of the $(\mathrm{O}-\mathrm{C})$ residuals suggested the presence of a circumbinary planet of $\sim 12 M_{J}$, but with an uncertain period and high eccentricity. Almeida et al. (2012) provided light curve and spectroscopic analysis whilst comparing NSVS 14256825 with nine other sdB binary systems. Almeida et al. (2013) presented ten new eclipse times obtained between 2010 July and 2012 August. They also combined their data with previous published measurements and performed a new orbital period analysis. They interpreted the observed eclipse timing variations as being the result of LTT effects introduced by two circumbinary planets with orbital periods of 3.5 and $6.9 \mathrm{yr}$ and masses $3 M_{J}$ and $8 M_{J}$, respectively.

Hinse et al. (2014) questioned the validity of these results. While searching extensively for a minimum in residuals, their 
approach predicted the orbital parameters and minimum mass of a single circumbinary companion. They performed two independent analyses based on two datasets (i) Dataset I as presented in Table 3 of Almeida et al. (2013) spanning an observing baseline of around $5 \mathrm{yr}$ and (ii) Dataset II, which is like Dataset I but with three earlier primary eclipse data points from Beuermann et al. (2012) extending the observing baseline to approximately $13 \mathrm{yr}$, so more than doubling the time window. With Dataset I they derived parameters similar to Almeida, but concluded that this was a local minimum and that a much longer baseline of data was needed to confirm this hypothesis. They found no evidence for a second companion. From Dataset II they derived a much longer cyclical period of 102 yr for a circumbinary object, but again concluded that the baseline was inadequate to draw firm conclusions. From their extensive statistical analysis they showed that the data did not constrain any particular model with wellestablished confidence limits.

Wittenmyer et al. (2013) also challenged the two-planet hypothesis from the standpoint of orbital stability where they concluded that the planetary system would have a life expectancy of less than $1000 \mathrm{yr}$.

More recently Nasiroglu et al. (2017) provided 83 new eclipse timings spanning the period 2009 August to 2016 November. Including earlier data, they analysed three datasets and reported that their Dataset $\mathrm{C}$, which excluded data from NSVS, the All Sky Automatic Survey (ASAS), and SuperWASP, indicated a possible brown dwarf circumbinary companion.

\subsubsection{Recent data and ephemeris}

We have added 19 new primary minima observed between 2015 August and 2017 July. Our new data, together with that from Nasiroglu et al. (2017) allowed us to test the circumbinary stellar object hypothesis of Almeida et al. (2013). We did not test the claims made by Qian et al. (2010), who have yet to publish their parameters, nor have we tested the findings of Beuermann et al. (2012) and Hinse et al. (2014), who both recognised that their derived circumbinary parameters were poorly constrained.

To investigate further the Almeida et al. (2013) hypothesis we use their ephemeris:

$T_{\min , \mathrm{BJD}}=2454274.20874(4)+0.1103741681(5) * E$.

From this linear ephemeris and observational data we calculate the RMS of the weighted residuals to be 20.95. Using our new data and all available historic data we have plotted the (O-C) residuals shown in Fig. 9 and have included predictions of Almeida's two-planet hypothesis. We find that from 2013 March $(E \sim 18900)$ calculated residuals depart significantly from Almeida's predictions increasing to $160 \mathrm{~s}$ at $E \sim 30000$.

Nasiroglu et al. (2017) proposed an alternative solution of a single circumbinary brown dwarf companion of mass $14 M_{J}$ with a 9.7-yr orbital period and 0.2 eccentricity. However, they noted that their new parameters "substantially differ from previous estimates". Using their linear ephemeris, Eq. (11), we find our new data are consistent with their Dataset $\mathrm{C}$ as shown in Fig. 10.

$T_{\min , \mathrm{BJD}}=2455793.84005(3)+0.110374099(3) * E$.

We tested statistically the fit between the $(\mathrm{O}-\mathrm{C})$ residuals and Nasiroglu's single circumbinary brown dwarf hypothesis and found the fit is significant at the $3 \sigma$ level. We conclude that the current data supports the Nasiroglu hypothesis, but caution that the data only spans one circumbinary cycle and confidence in this model will require a significantly longer timebase.

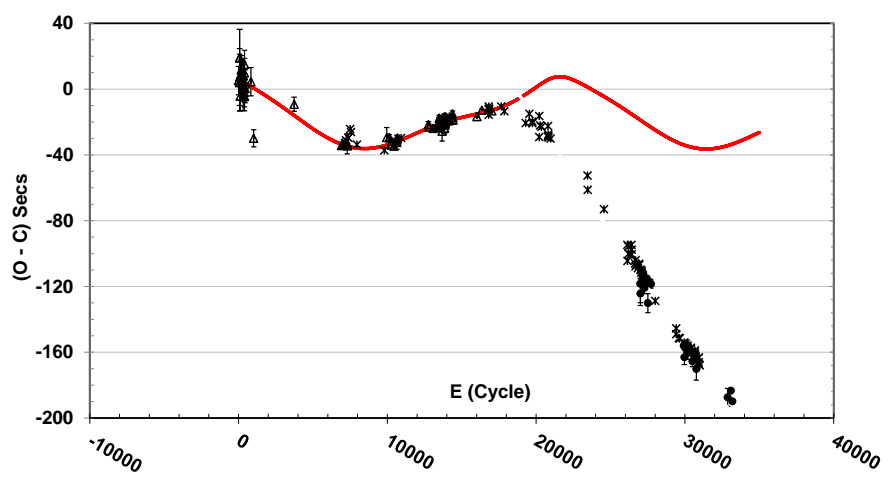

Fig. 9. Residuals for NSVS 14256825 calculated from the ephemeris and orbital data from Almeida (2013). Open triangles represent data from Almeida; stars represent data from Nasiroglu (for clarity error bars are not shown); our new data are shown as solid circles. The solid line represents Almeida's two-planet prediction showing departure at $E \sim 18900$.

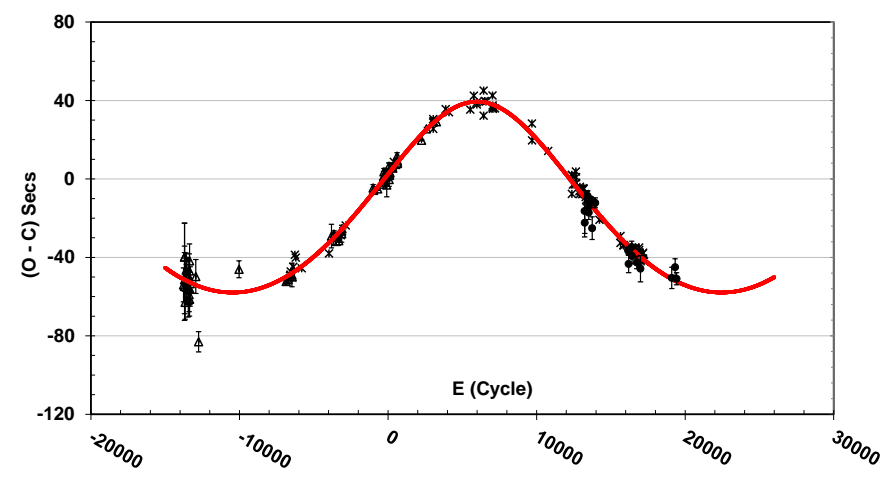

Fig. 10. Residuals for NSVS 14256825 calculated from the ephemeris and orbital data from Nasiroglu (2017). Open triangles represent data from Almeida; the stars represents data from Nasiroglu; our new data are shown as solid circles. The solid line represents Nasiroglu's circumbinary brown dwarf hypothesis.

\subsection{NY Vir}

\subsubsection{Background}

From spectroscopic and light curve analysis, Kilkenny et al. (1998) first reported NY Vir (PG1336-018) as a detached short period, $\sim 2.4 \mathrm{~h}$, eclipsing binary consisting of a pulsating hot sdB star and a probable dwarf M5 companion. From their observations, including ten times of minima observed between 1996 May and 1996 July, they computed a linear ephemeris with binary period of 0.1010174 days. Kilkenny (2011) published a further 12 times of minima extending the timeline to 2010 June and noted that the eclipse times departed significantly from those predicted by a linear ephemeris. With their new data, they showed that the period was rapidly changing, fitting a quadratic ephemeris to the $(\mathrm{O}-\mathrm{C})$ residuals and noting that, due to the sdB pulsations, the eclipse timings were less accurate than would normally be expected. Çamurdan et al. (2012) found a similar effect, publishing a further 7 times of minima between 2009 May and 2011 June and suggesting a possible LTT effect caused by the presence of a third body.

A further nine times of minima were added by Qian et al. (2012) who favoured a quadratic ephemeris with a third body contributing a LTT effect of amplitude $6.3 \mathrm{~s}$, a period of $7.9 \mathrm{yr}$, and mass greater than $2 M_{J}$. They also suggested that the rapid 
period reduction could be part of a long-term effect from a fourth body. Lee et al. (2014) provided a further 39 times of minima between 2011 January and 2014 May. They also included four earlier weighted mean datasets from Vucković et al. (2007) and their analysis agreed well with that of Qian et al. (2012) indicating a third body of period $8.18 \mathrm{yr}$, a mass of $2.78 \mathrm{M}_{\mathrm{J}}$, and an LTT effect of $6.9 \mathrm{~s}$. They also postulated a fourth planetary body of period $27.0 \mathrm{yr}$, mass of $4.49 M_{J}$, and LTT effect of $27.3 \mathrm{~s}$ to explain the rapid period reduction. However, with a predicted orbital eccentricity of zero the orbital stability of this four-body system was calculated to be only $800000 \mathrm{yr}$. As a consequence they suggested that a longer timebase was required to substantiate the presence of the fourth body and that it should have a moderate eccentricity to bring long-term orbital stability.

\subsubsection{Recent data and ephemeris}

We added a further 15 times of primary minima observed between 2015 April and 2017 June extending the timebase by $3 \mathrm{yr}$. In addition, we have added four times of minima extracted from the database of the AAVSO of which two data points fall within the latter part of the data range of Lee et al. (2014) and two within the range of our new data. We employed the same criteria for data selection as used by Lee taking only primary minima that had uncertainties of less than 0.0001 days. We investigated the secondary minima, but found they gave a large degree of scatter about the predicted trend line. This was most probably due to the amplitude of the pulsations being comparable to the depth of the secondary minima (see e.g. Kilkenny et al. 1998, Fig. 3).

To enable us to make a direct comparison with the Lee et al. (2014) two-planet prediction we used their linear ephemeris.

$T_{\min , \mathrm{BJD}}=2453174.442699(91)+0.1010159668(43) * E$

The calculated RMS of the weighted residuals was found to be 36.45. Examining the data we find that the uncertainties associated with Vuckovic's two primary minima were much lower than the remaining 75 uncertainties. These two uncertainties were found to be significantly biasing the RMS value and, after removing them, the RMS of weighted residuals decreased to 4.78.

Figure 11 shows our new data points relative to Lee's twoplanet hypothesis. Our new data show that the eclipses occur between $10 \mathrm{~s}$ and $20 \mathrm{~s}$ before those anticipated by Lee's model, whilst the four AAVSO data points $(E=27769,27770$, 39449 , and 42934) link our new data with the prediction from Lee. Kilkenny (2014) also indicates a departure from Lee's predictions.

\section{Discussion}

\section{1. $s d B$ binaries: 20 yr of eclipse time data}

The last decade has seen many predictions made for the existence of circumbinary companions orbiting post-common envelope eclipsing sdB binary systems. It was found that these objects were typically greater than several Jupiter masses and with orbital periods of between 3 to $30 \mathrm{yr}$. We have reviewed seven of these systems, of which five (HS 0705+6700, HS 2231+2441, NSVS 07826147, NSVS 14256825, and NY Vir) have previously been reported as most probably having circumbinary companions. Of the remaining two systems, EC 10246-2707 has $20 \mathrm{yr}$ of observational data but shows no clear indication of cyclical LTT effects. The second system, J08205+0008, has five years of data, and though there is little evidence of LTT effects,

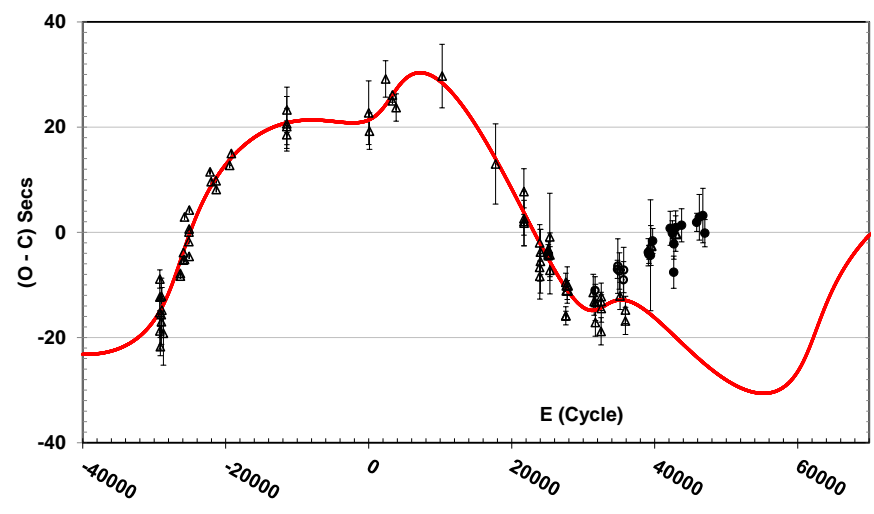

Fig. 11. NY Vir residuals using Lee's linear ephemeris plus two planet hypothesis. Early data from Kilkenny, Lee, Camurdan and AAVSO are shown as open triangles; our new data (solid circles) and Kilkenny's more recent data (open circles) show departure from Lee's two-planet hypothesis (solid line).

it would be premature to draw any firm conclusions about the presence, or absence, of a circumbinary companion.

Of the five systems reported as possibly having circumbinary companions three, HS 0705+6700, NSVS 14256825, and NY Vir, have recently shown significant changes in their predicted eclipse times. These changes might indicate that a longer time baseline is needed to validate the circumbinary hypothesis, but could also suggest that other mechanisms, e.g. magnetic effects, are driving the observed eclipse time variations.

In early 2013 NY Vir began to show a departure from the predictions of Lee et al. (2014). These changes were noted by both Kilkenny (2014) and our team (Pulley et al. 2016a). Our new data do not rule out the possibility of circumbinary planets, but they do raise questions as to whether the $21 \mathrm{yr}$ of observations is sufficient to base predictions on.

NSVS 14256825 also provides conflicting evidence for the circumbinary hypothesis. Almeida et al. (2013) proposed a twocircumbinary solution with periods of 3.5 and $6.7 \mathrm{yr}$, respectively, which has been challenged by Hinse et al. (2014) and others and the latest observations no longer support the Almeida model. Single circumbinary solutions have been put forward by Beuermann et al. (2012) suggesting a broad range of possible periods extending from 14 to $30 \mathrm{yr}$ and Hinse et al. (2014) who preferred a period of either 2.5 or $6 \mathrm{yr}$, depending upon the dataset used. Qian et al. (2009) also recognised a periodicity in the data, but they have not yet specified a value for the period. In their review of this object, Nasiroglu et al. (2017) computed new circumbinary parameters and remaining open minded about its presence; they predicted a possible circumbinary brown dwarf with a period of $9.7 \mathrm{yr}$. Future observations will either support the circumbinary hypothesis or provide evidence against it. In any case, longer baselines covering full orbits of potential circumbinary objects are clearly needed.

HS $0705+6700$ provides yet another example of conflicting evidence for the circumbinary companion hypothesis. Data covering more than $14 \mathrm{yr}$ have shown apparent periodicity in its eclipse timings with most observers agreeing on a circumbinary period of between 7.2 and $8.9 \mathrm{yr}$. Whilst providing nearly two orbits of data, and as confidence grew in this model, in late 2015 this system ceased exhibiting its apparently cyclical LTT behaviour and now shows an apparently constant period.

Of the remaining two systems with circumbinary companion claims, HS $2231+2441$ is somewhat different. Whilst one 
investigator suggested a circumbinary object of period $4.76 \mathrm{yr}$ we find that the Lohr et al. (2014) suggestion of a constant period and no significant LTT effects as more consistent with the longer term observations. Our new data extends the timebase to approximately $12 \mathrm{yr}$ and we find little convincing evidence of cyclical LTT effects and suggest a revised constant period of 0.110587855 days.

Finally, only one investigator, Zhu et al. (2015a), has detected a possible circumbinary companion to the $\mathrm{sdB}$ system NSVS 07826147. Although they have published little on this, their diagrams suggest a period of about $4.9 \mathrm{yr}$ with an LTT amplitude of $3.5 \mathrm{~s}$. This amplitude is small, and could be considered within observational noise. Moreover, our new data observed through 2015 and 2016 provides no clear evidence to support their circumbinary claims.

\subsection{A changing landscape}

While some authors have challenged specific details of the circumbinary planet hypotheses, others have challenged the very existence of such planets around close binaries. Wittenmyer et al. (2013) challenged Almeida's two-planet hypotheses for the binary system NSVS 14256825, whilst Horner et al. (2012) challenged the two-planet model proposed for the HW Vir system. Again, from an orbital stability standpoint, Horner et al. (2014) challenged the two-planet systems of three other binaries. Hardy et al. (2015), using the new extremeAO instrument SPHERE, imaged the prototype eclipsing postcommon envelope binary V471 Tau in search of a brown dwarf that was predicted to be responsible for LTT effects. They found no direct evidence of such an object. More recently, Bours et al. (2016) carried out a long-term programme of eclipse time measurements on 67 white dwarfs in close binaries to detect period variations. They found that all systems with baselines exceeding $10 \mathrm{yr}$, and with companions of spectral type M5 or earlier, appeared to show much greater eclipse timing variations than systems with companions of spectral types later than M5. They found this to be consistent with an Applegate-type mechanism. Nevertheless, they also considered it reasonable to assume that some planetary systems could exist around evolved white dwarf binaries, for example the NN Ser binary system (Beuermann et al. 2013; Marsh et al. 2013; Parsons et al. 2014; Hardy et al. 2016).

\subsection{Statistical trends of sdB eclipse time variations}

Following the methodology of Bours et al. (2016), we investigated the correlation between the RMS residuals of the seven sdB binary systems reported here with their observational baselines and their spectral types. Unfortunately, the spectral types of the binary companions are somewhat uncertain as these stars are not spectroscopically visible and assessment has to be made indirectly through parameters that are generally poorly constrained. Spectral types have not been published for HS 0705+6700 and NSVS 14256825, but we have derived their values from their calculated secondary mass in conjunction with Baraffe \& Chabrier (1996). Spectral types and RMS values are summarised in Table 2.

Bours concluded that an observational baseline of at least $10 \mathrm{yr}$ was necessary to show RMS values saturating at around 100 and all white dwarf $+M$ dwarf binaries considered showed significant $(\mathrm{O}-\mathrm{C})$ residuals of the order of $100 \mathrm{~s}$. Our observations are summarised in Fig. 12, where six of the seven systems have more than $10 \mathrm{yr}$ observing time, but we find none



Fig. 12. Calculated RMS of residuals as a function of observing period. Only J08205+008 has an observing period of less than $10 \mathrm{yr}$ and as yet shows little LTT effect. All of these systems fall short of the Bours et al. (2016) criteria of RMS 100 .

of these systems approaching the RMS values of 100. Two systems, HS 0705+6700 and NSVS 14256825, showed strong LTT effects but with RMS values of only about 20 . We found four of the systems having an RMS value of less than 5 including one, NY Vir after removing the two Vuckovic data points, showing definite LTT effects. Only HS 0705+6700 showed large (O-C) residuals at the $100 \mathrm{~s}$ level suggested by Bours. The remaining two systems, NSVS 14256825 and NY Vir were much less at $50 \mathrm{~s}$ and $25 \mathrm{~s}$, respectively.

To achieve a Bours RMS saturation figure of $~ 100$, Eq. (3) suggests large cyclical LTT amplitudes ( $>100 \mathrm{~s}$ ) and/or small uncertainties $(<0.00004$ days $)$ are necessary. The high values of RMS found by Bours could possibly be attributed to the observational equipment used which will tend to give lower values of uncertainties. Historical observations of sdB binaries typically used much smaller telescopes $(<1 \mathrm{~m})$ and less sophisticated cameras yielding typical uncertainties an order of magnitude, or more, higher.

We also compared the RMS of weighted residuals with the spectral type of the sdB companion (see Fig. 13). As noted earlier, the methodology used to determine the spectral type of the secondary component results in values with a high degree of uncertainty. Of particular note are EC 10246-2707 and NSVS 07826147, which both show a low RMS and (O-C) values, but are classified as M5 and are potentially magnetically active. This may indicate a spectral type misclassification or possibly both subdwarfs showing no magnetic activity. The three systems exhibiting significant LTT effects, HS 0705+6700, NSVS 14256825 , and NY Vir, appear to be possible M6 or earlier and are in agreement with the findings of Bours.

Whilst the sdB data are not in complete agreement with the Bours approach, there is still the need to identify those systems which show real LTT effects from those that show ETVs more likely caused by random measurement uncertainties. Using an unweighted RMS statistic, i.e. setting sigma equal to unity in Eq. (3), we can compare the mean $1 \sigma$ uncertainty levels of each system with their corresponding $(\mathrm{O}-\mathrm{C})$ residuals taken from a linear or quadratic ephemeris baseline. These results are summarised in Table 4 where three systems, HS 0705+6700, NSVS 14256825 , and NY Vir show greater than $3 \sigma$ variations due to LTT effects, indicating the possible presence of some physical phenomena, such as circumbinary or magnetic effects. Four systems do not meet the $3 \sigma$ criteria where two, EC 10246-2707 and J08205+0005, show LTT effects at the $\sim 1 \sigma$ level suggesting there is unlikely to be any physical activity in these systems. The 
D. Pulley et al.: The quest for stable circumbinary companions to post-common envelope sdB eclipsing binaries

Table 4. Comparison of the LTT effect from a linear or quadratic baseline with $1 \sigma$ uncertainty levels.

\begin{tabular}{lcccccc}
\hline \hline Object & $\begin{array}{c}\text { Measurement } \\
\text { uncertainty } \\
\text { (days) }\end{array}$ & $\begin{array}{c}\text { (O-C) Lin } \\
\text { (days) }\end{array}$ & $\begin{array}{c}\text { (O-C) Quad } \\
\text { (days) }\end{array}$ & $\begin{array}{c}\text { No of } \sigma \\
\text { (lin) }\end{array}$ & $\begin{array}{c}\text { No of } \sigma \\
\text { (quad) }\end{array}$ & $\begin{array}{c}\text { Bours } \\
\text { weighted } \\
\text { RMS }\end{array}$ \\
\hline EC 10246-2707 & 0.0000846 & 0.0000983 & 0.0000800 & 1.16 & 0.95 & 1.60 \\
HS 0705+6700 & 0.0001263 & & 0.0007412 & & 5.87 & 19.89 \\
HS 2231+2441 & 0.0001671 & 0.0004178 & 0.0004031 & 2.50 & 2.41 & 2.47 \\
J08205+0008 & 0.0001256 & 0.0001177 & 0.0001140 & 0.94 & 0.91 & 1.13 \\
NSVS 07826147 & 0.0000542 & 0.0001155 & 0.0001144 & 2.13 & 2.11 & 2.41 \\
NSVS 14256825 & 0.0000387 & 0.0003867 & & 10.0 & & 20.95 \\
NY Vir & 0.0000313 & 0.0001413 & & 4.51 & & $4.78 /$ \\
& & & & & & 36.45 \\
\hline
\end{tabular}

Notes. The Bours' weighted statistic is shown for comparison where NY Vir shows two values, one for the exclusion and the other for the inclusion of Zorotovic's two ULTRACAM low uncertainty observations.

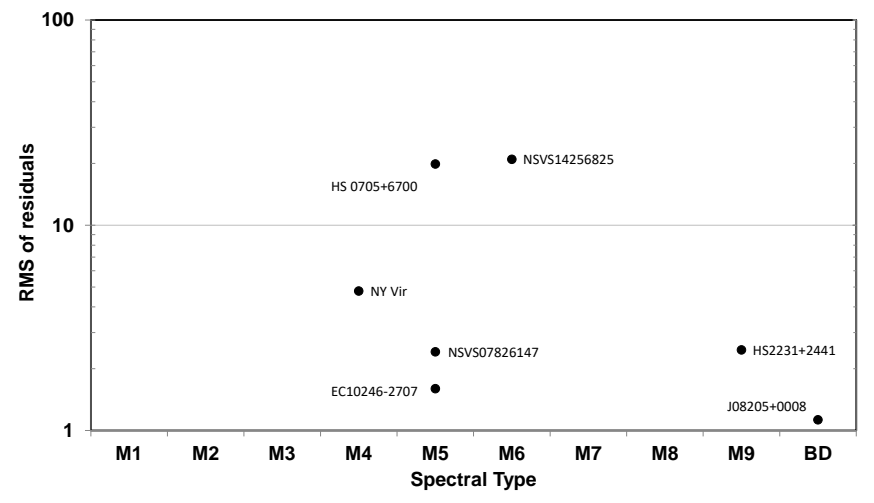

Fig. 13. RMS of residuals with respect to spectral type. There is some uncertainty of spectral type for all of these systems. Of interest are EC 10246-2707 and NSVS 07826147, classified as M5 but exhibiting little or no LTT effect over $15 \mathrm{yr}$ or more of observation.

remaining systems, HS 2231+2441 and NSVS 07826147, show LTT effects at the $2 \sigma$ to $2.5 \sigma$ level, possibly indicating some form of low level activity. For comparison purposes, the Bours weighted RMS statistic is included in Table 4, which follows the same trend as our analysis.

\subsection{Possible circumbinary companion transits}

It is conceivable that circumbinary companions may be detected transiting these binary systems especially if their orbital plane is closely aligned with the orbital plane of the binary system. The current predicted circumbinary companions have orbital periods measured in years and orbital distances in AU giving them a low probability of making a detectable transit. However, close-in circumbinary companions with orbital periods measured in days, and possibly in orbital resonance with the binary, might well make transits that could be detected in the system's light curve. The expected impact parameter of such transits is expected to be low given that the orbital plane of the binary is closely aligned with the observer's line of sight. In general, the best eclipsing binaries to observe would be the less massive binaries. For such binaries transiting planets dim a greater fraction of their light than for more massive binaries.

Whether such companions exist is speculative, but there have been several occasions, and on different binary systems, where we recorded transit-like dimming in the systems light curve. First observed in 2013 December, the duration and depth of the dip looked very similar to a circumbinary transit. More sightings will be needed before we can make and test predictions.

\section{Conclusions}

The complex energy interaction of detached short period $\mathrm{sdB}+$ M dwarf/brown dwarf binary systems makes these systems difficult to model with best fit solutions often requiring the secondary component's albedo to take on non-physical values of greater than unity (see e.g. Drechsel et al. 2001). These difficulties are compounded by the secondary star not being spectroscopically visible. Also, the formation of circumbinary objects remains an open question. If they do exist, were they formed before or after the common envelope ejection, or a combination of both (see e.g. Schleicher \& Dreizler 2014)?

These uncertainties can make it difficult to interpret observed ETVs, particularly when timelines are short. Our recent observations of seven sdB binary systems, together with historical data, indicate the following:

From earlier eclipse time observations, it has been suggested that five of the seven systems (HS 0705+6700, HS 2231+2441, NSVS 07826147, NSVS 14256825, and NY Vir) reported here most probably had circumbinary companions. More recent observations indicate only three of these systems (HS 0705+6700, NSVS 14256825 and NY Vir) show significant LTT effects. All three have recently shown very significant changes from their earlier circumbinary hypothesis. HS $0705+6700$, after more than 1.5 circumbinary periods, now gives little indication of ETVs. It is evident that observation of several consecutive and consistent LTT cycles is a prerequisite to support a third body hypothesis.

Whilst NY Vir and NSVS 14256825 have shown significant changes from earlier eclipse time predictions, both still show a cyclical shape to their $(\mathrm{O}-\mathrm{C})$ residuals. In particular the residuals of NSVS 14256825 give a very close fit to the more recent predicted parameters of Nasiroglu et al. (2017). However, both systems report only one cycle of a proposed circumbinary companion and drawing firm conclusions would thus be premature.

Four systems (EC 10246-2707, HS 2231+2441, J0802+0008, and NSVS 07826147) show little or no ETV. Linear and quadratic ephemerides have been determined for each system and although all four systems tended to give marginally lower residuals with the quadratic ephemeris, statistically there was no significant difference between either ephemeris.

We find agreement with Bours et al. (2016) that a decade or so of observations are required to establish a reliable ephemeris, but we do not find a tendency for the RMS weighted residuals to saturate at $\sim 100$. For the systems 
we considered we found RMS values ranging between 1.6 and 21.0. We also found only one of the seven systems (HS 0705+6700) had $(\mathrm{O}-\mathrm{C})$ residuals approaching $100 \mathrm{~s}$; the other systems fall significantly short of this target. We found that the RMS value was strongly dependent upon the LTT amplitude and inversely dependent upon the uncertainty in minima. Systems with a few abnormally low uncertainty values can significantly bias upwards the magnitude of the RMS weighted residuals.

Although the spectral types of sdB companions are not tightly constrained, we are in general agreement with Bours that larger values of RMS of residuals are generally found with companions of spectral type M5/6 or earlier. This is the boundary where a star becomes fully convective and may be indicative that significant LTT effects are being driven by magnetic processes within the companion star, possibly from a modified Applegate-type mechanism. There are some possible anomalies (e.g. EC 10246-2707 and NSVS 07826147), which are both classified as M5 but show few LTT effects.

A longer time baseline is required to resolve underlying nature of these ETVs, but so far only NY Vir has been included in the NASA Exoplanet archive. Similarly, for short period white dwarf eclipsing binaries only NN Ser, DP Leo, RR Cae, and UZ For have confirmed exoplanets.

Acknowledgements. This work was partly funded by the British Astronomical Association under BAA account BAAHS0705. This work also makes use of observations from the LCO network of telescopes and of the APASS database maintained on the AAVSO website. We would like to thank Dr. Marcus Loh (The Open University) who provided information on the methodology used in analysing the SuperWASP data and Dr. David Boyd for providing two sets of observations that enabled us to link our new data with the historical observations of NY Vir. We would also like to thank Dr. Brad Barlow (High Point University), Dr. Klaus Beuermann (University of Goettingen), Dr. Roy Østensen (University of Leuven), Dr. Stephan Geier (University of Tübingen), Dr Veronika Schaffenroth (University of Innsbruck), Patrick Wils (Vereniging voor Sterrenkunde), Dr Slowikowska (University of Zielona Góra), and Dr. S-B Qian (Chinese Academy of Sciences) who addressed many of the questions we posed. We would also like to thank the referee whose guidance was greatly appreciated.

\section{References}

Almeida, L. A., Jablonski, F., Tello, J., \& Rodrigues, C. V. 2012, MNRAS, 423 478

Almeida, L. A., Jablonski, F., \& Rodrigues, C. V. 2013, ApJ, 766, 11

Almeida, L. A., Damineli, A., Jablonski, F., Rodrigues, C., \& Cieslinski, D.

2014, Rev. Mex. Astron. Astrofis. Conf. Ser., 44, 35

Applegate, J. H. 1992, ApJ, 385, 621

Backhaus, U., Bauer, S., Beuermann, K., et al. 2012, A\&A, 538, A84

Baraffe, I., \& Chabrier, G. 1996, ApJ, 461, L51

Barlow, B., Kilkenny, D., Drechsel, H., et al. 2013, MNRAS, 430, 22

Bear, E., \& Soker, N. 2014, MNRAS, 444, 1698

Beuermann, K., Breitenstein, P., Debski, B., et al. 2012, A\&A, 540, A8

Beuermann, K., Dreizler, S., \& Hessman, F. V. 2013, A\&A, 555, A133

Bours, M. C., Marsh, T., Parsons, S., et al. 2016, MNRAS, 460, 3873

Brinkworth, C., Marsh, T., Dhillon, V., \& Knigge, C. 2006, MNRAS, 365, 287
Çamurdan, C. M., Zengin Çamurdan, D., \& Ibanoglu, C. 2012, New Astron., 17, 325

Drake, A., Beshore, E., Catelan, M., et al. 2010, ArXiv e-prints [arXiv: 1009.3048v1]

Drechsel, H., Heber, U., Napiwotzki, R., et al. 2001, A\&A, 379, 893

For, B.-Q., Green, E., Fontaine, G., et al. 2010, ApJ, 708, 253

Geier, S., Schaffenroth, V., Drechsel, H., et al. 2011, ApJ, 731, L22

Geier, S., Classen, L., Brünner, P., et al. 2012, ASP Conf. Ser., 452, 153

Hardy, A., Schreiber, M., Parsons, S., et al. 2015, ApJ, 800, L24

Hardy, A., Schreiber, M. R., Parsons, S. G., et al. 2016, MNRAS, 459, 4518

Hinse, T. C., Lee, J. W., Goździewski, K., Horner, J., \& Wittenmyer, R. A. 2014 MNRAS, 438, 307

Horner, J., Hinse, T., Wittenmyer, R., Marshall, J., \& Tinney, C. 2012, MNRAS, 427, 2812

Horner, J., Wittenmyer, R., Marshall, J., Hinse, T., \& Robertson, P. 2014, Astron. Geophys., 55, 4

Irwin, J. B. 1952, ApJ, 116, 211

Kelley, N., \& Shaw, J. 2007, J. Southeastern Assoc. Res. Astron., 1, 13

Kilkenny, D. 2011, MNRAS, 412, 487

Kilkenny, D. 2014, MNRAS, 445, 4247

Kilkenny, D., \& Koen, C. 2012, MNRAS, 421, 3238

Kilkenny, D., O’Donoghue, D., Koen, C., Stobie, R., \& Chen, A. 1997, MNRAS, 287,867

Kilkenny, D., O’Donoghue, D., Koen, C., Lynas-Gray, A., \& van Wyk, F. 1998, MNRAS, 296, 329

Kwee, K. K., \& van Woerden, H. 1956, Bull. Astron. Inst. Neth., 12, 327

Lee, J. W., Hinse, T. C., Youn, J.-H., \& Han, W. 2014, MNRAS, 445, 2331

Lohr, M. E., Norton, A. J., Anderson, D. R., et al. 2014, A\&A, 566, A128

Marsh, T. R., Parsons, S., Bours, M. C., et al. 2013, MNRAS, 437, 475

Nasiroglu, I., Goździewski, K., Słowikowska, A., et al. 2017, AJ, 153, 137

Niarchos, P., Gazeas, K., \& Manimanis, V. 2003, in Interplay of Periodic, Cyclic and Stochastic Variability in Selected Areas of the HR Diagram, 292, 129

Østensen, R., Oreiro, R., Drechsel, H., et al. 2007, ASP Conf. Ser., 372, 483

Østensen, R., Oreiro, R., Hu, H., Drechsel, H., \& Heber, U. 2008, ASP Conf Ser, 392, 221

Paczynski, B. 1976, in Symposium-International Astronomical Union (Cambridge Univ. Press), 73, 75

Parsons, S. G., Marsh, T., Bours, M. C., et al. 2014, MNRAS, 438, L91

Pulley, D., Faillace, G., Smith, D., Watkins, A., \& Owen, C. 2015, J. Brit. Astron. Assoc., 125, 5

Pulley, D., Faillace, G., Smith, D., \& Watkins, A. 2016a, J. Brit. Astron. Assoc., 126,4

Pulley, D., Smith, D., Faillace, G., \& Watkins, A. 2016b, J. Brit. Astron. Assoc., 126, 2

Qian, S.-B., Zhu, L.-Y., Zola, S., et al. 2009, ApJ, 695, L163

Qian, S.-B, Zhu, L., Liu, L., et al. 2010, Astrophys. Space Sci., 329, 113

Qian, S.-B., Zhu, L.-Y., Dai, Z.-B., et al. 2012, ApJ, 745, L23

Qian, S.-B., Shi, G., Zola, S., et al. 2013, MNRAS, 436, 1408

Schaffenroth, V., Geier, S., Heber, U., et al. 2011, AIP Conf. Proc., 1331, 174

Schleicher, D. R., \& Dreizler, S. 2014, A\&A, 563, A61

Vucković, M., Aerts, C., Østensen, R., et al. 2007, A\&A, 471, 605

Webbink, R. 1984, ApJ, 277, 355

Wils, P., di Scala, G., \& Otero, S. A. 2007, IBVS, 5800, 1

Wittenmyer, R. A., Horner, J., \& Marshall, J. P. 2013, MNRAS, 431, 2150

Woźniak, P., Vestrand, W., Akerlof, C., et al. 2004, AJ, 127, 2436

Xiong, H., Chen, X., Podsiadlowski, P., Li, Y., \& Han, Z. 2017, A\&A, 599, A54

Zhu, L., \& Qian, S. 2010, Astrophys. Space Sci., 329, 107

Zhu, L. Y., Qian, S. B., Liao, W. P., et al. 2015a, Kor. Pub. Astron. Soc., 30, 289

Zhu, L. Y., Qian, S. B., Zhao, E.-G., Lajús, E. F., \& Han, Z.-T. 2015b, Acta Polytechnica CTU Proceedings, 2, 183

Zorotovic, M., \& Schreiber, M. R. 2013, A\&A, 549, A95

Zorotovic, M., Schreiber, M. R., Gaensicke, B. T., \& Gómez-Morán, A. N. 2010, A\&A, 520, A86 
D. Pulley et al.: The quest for stable circumbinary companions to post-common envelope sdB eclipsing binaries

\section{Appendix A: Tables at the CDS}

Table A.1. Telescopes and instrumentation used for the measurements reported in this paper.

\begin{tabular}{llll}
\hline \hline Observatory & Telescope & Instrumentation & MPC Code \\
\hline Sierra Stars Observatory Network & $0.61 \mathrm{~m} \mathrm{~F} / 10$ Optical & Finger Lakes Inst & G68 \\
Markleeville & Mechanics & ProLine camera & \\
California, USA & Nighthawk CC06 & $3056 \times 3056$ pixels & \\
http://sierrastars.com/gp/SSO/SSO--CA.aspx & & FOV 21 × 21 arcmin \\
\hline
\end{tabular}

Notes. The full table is available at the CDS.

Table A.2. Compilation of our new measurements observed between 2013 September and 2017 July.

\begin{tabular}{lcccll}
\hline \hline BJD & $\begin{array}{c}\text { Error } \\
\text { (days) }\end{array}$ & Cycle & Minima & Filter & Telescope \\
\hline EC 10246-2707 & $T_{0}=2450493.46733$ & & & & \\
\hline 2457407.697910 & 0.000019 & 58344 & $I$ & $V$ Bessell & 1 m Cerro Tololo, Chile, LCO \\
2457408.645870 & - & 58352 & $I$ & $B$ Bessell & 1 m Cerro Tololo, Chile, LCO \\
2457425.592548 & 0.000015 & 58495 & $I$ & $V$ Bessell & 1 m Sutherland, SA, LCO \\
2457436.139746 & 0.000088 & 58584 & $I$ & Sloan $r^{\prime}$ & 0.43 m iTelescope, Siding Spring T17 \\
2457449.531205 & 0.000022 & 58697 & $I$ & Unfiltered & 1 m Sutherland, SA, LCO \\
2457775.072622 & 0.000091 & 61444 & $I$ & Sloan $r^{\prime}$ & 0.43 m iTelescope, Siding Spring T17 \\
2457777.087370 & 0.000084 & 61461 & $I$ & Sloan $r^{\prime}$ & 0.43 m iTelescope, Siding Spring T17 \\
\hline HS 0705+6700 & $T_{0}=2451822.76155$ & & & \\
\hline
\end{tabular}

Notes. The reference epoch is noted for each binary system in each section header. The full table is available at the CDS.

Table A.3. Coordinates and apparent magnitudes of comparison stars from the APASS catalogue.

\begin{tabular}{lccccccccc}
\hline \hline $\begin{array}{l}\text { Binary } \\
\text { system }\end{array}$ & $\begin{array}{c}\text { Star } \\
\text { Ref No }\end{array}$ & Johnson $V$ & Johnson $B$ & Sloan $g^{\prime}$ & Sloan $r^{\prime}$ & Sloan $i^{\prime}$ & RA & Dec & $\begin{array}{c}\text { Distance from } \\
\text { target (arcmin) }\end{array}$ \\
\hline EC 10246-2707 & 1 & 13.273 & 13.699 & 13.443 & 13.158 & 13.079 & $10: 27: 02.841$ & $-27: 21: 02.57$ & 2.5 \\
RA 10:26:56.472 & 2 & 14.220 & 14.750 & 14.427 & 14.052 & 13.943 & $10: 26: 57.964$ & $-27: 27: 27.17$ & 4.51 \\
Dec -27:22:57.11 & 4 & 13.460 & 13.898 & 13.604 & 13.271 & 13.179 & $10: 27: 14.648$ & $-27: 23: 28.54$ \\
& 5 & 14.701 & 15.253 & 14.914 & 14.521 & 14.352 & $10: 27: 07.271$ & $-27: 18: 48.15$ \\
& 6 & 15.225 & 15.795 & 15.506 & 15.153 & 15.038 & $10: 27: 13.378$ & $-27: 16: 18.34$ \\
& 7 & 14.500 & 15.166 & 14.779 & 14.243 & 14.118 & $10: 26: 24.711$ & $-27: 18: 01.56$ & 7.86 \\
& 1 & 13.637 & 14.141 & 13.824 & 13.514 & 13.358 & $07: 11: 21.473$ & $67: 00: 57.55$ & 11.18 \\
\hline HS 0705+6700 & 2 & 13.435 & 14.172 & 13.728 & 13.190 & 12.967 & $07: 09: 10.503$ & $66: 58: 51.25$ & 23.08 \\
RA 07:10:42.056 & 3 & 13.798 & 14.230 & 13.947 & 13.685 & 13.574 & $07: 09: 24.091$ & $67: 01: 45.42$ & 20.38 \\
Dec 66:55:43.52 & 4 & 13.842 & 14.284 & 14.001 & 13.757 & 13.636 & $07: 10: 24.685$ & $66: 56: 18.49$ & 4.36 \\
& 6 & 14.135 & 14.488 & 14.239 & 14.047 & 13.946 & $07: 09: 47.389$ & $66: 55: 16.33$ & 13.65 \\
\hline
\end{tabular}

Notes. The star reference numbers refer to star charts used for this work. The full table is available at the CDS. 